ICCUB-18-020

\title{
Late-time Cosmic Acceleration from Compactification
}

\author{
J. G. Russo ${ }^{a, b}$ and P. K. Townsend ${ }^{c}$ \\ a Institució Catalana de Recerca i Estudis Avançats (ICREA), \\ Pg. Lluis Companys, 23, 08010 Barcelona, Spain. \\ b Departament de Física Cuántica i Astrofísica and Institut de Ciències del Cosmos, \\ Universitat de Barcelona, Martí Franquès, 1, 08028 Barcelona, Spain. \\ c Department of Applied Mathematics and Theoretical Physics, \\ Centre for Mathematical Sciences, University of Cambridge, \\ Wilberforce Road, Cambridge, CB3 0WA, UK. \\ E-Mail: jorge.russo@icrea.cat, p.k.townsend@damtp.cam.ac.uk
}

\begin{abstract}
We investigate the implications of energy conditions on cosmological compactification solutions of the higher-dimensional Einstein field equations. It is known that the Strong Energy Condition forbids time-independent compactifications to de Sitter space but allows time-dependent compactifications to other (homogeneous and isotropic) expanding universes that undergo a transient period of acceleration. Here we show that the same assumptions allow compactification to FLRW universes undergoing late-time accelerated expansion; the late-time stress tensor is a perfect fluid but with a lower bound on the pressure/energy-density ratio that excludes de Sitter but allows accelerated power-law expansion. The compact space undergoes a decelerating expansion that leads to decompactification, but on an arbitrarily long timescale.
\end{abstract}

Keywords: cosmology, compactification, energy conditions 


\section{Contents}

1 Introduction $\quad 2$

2 Warped compactifications to FLRW cosmologies 6

2.1 The Strong Energy Condition . . . . . . . . . . . . . . . . . . . 7

2.2 No-go theorems . . . . . . . . . . . . . . . . . . 8

2.3 Power-law acceleration . . . . . . . . . . . . . . . . . 9

2.4 Decompactification ..................... 11

2.5 SEC Redux . . . . . . . . . . . . . . . . . . . . . 12

3 The late-time stress tensor $\quad \mathbf{1 3}$

3.1 A new no-go theorem . . . . . . . . . . . . . . . . 15

3.2 The Dominant Energy Condition . . . . . . . . . . . . . . . . 16

3.3 Asymptotic zero acceleration . . . . . . . . . . . . . . . . 17

3.4 Generic Einstein metric for the compact space . . . . . . . . . . . . . . 18

4 Summary and discussion $\quad \mathbf{1 9}$

4.1 No-acceleration bounds on exponential scalar potentials . . . . . . . . . 20

\section{Introduction}

Observations indicating that the expansion of the Universe is accelerating are compatible with General Relativity (GR) if one postulates a dark energy contribution to the matter stress tensor. Equivalently, one may modify Einstein's field equations to include a positive cosmological constant, as originally proposed on other grounds by Einstein himself. Either way, the predicted late-time geometry of an expanding universe is that of de Sitter (dS) but difficulties facing any attempt to formulate a consistent quantum theory of gravity in de Sitter space [1] suggest that current observations may have some other explanation that does not lead to the prediction of a late-time dS universe.

In the context of scalar-tensor theories of gravity in which a $\mathrm{dS}$ vacuum corresponds to a minimum value $V_{0}>0$ of a scalar-field potential $V$, one can propose a function $V$ that has no such minima but does have regions in field space of positive $V$ with $|\nabla V|$ sufficiently small that accelerated expansion is both possible and sufficiently slowly varying to be compatible with current observations. Scalar fields that make this possible have been dubbed "quintessence". However, some of the difficulties facing the formulation of a consistent quantum theory of gravity in de Sitter space, in particular the future cosmological event horizon associated with late-time accelerated expansion, are still present in quintessence models [2, 3].

It is natural to ask what string/M-theory has to say about dS vacua and quintessence. In particular, one may ask whether the 10/11-dimensional supergravity theories that arise as low-energy effective field theories for string/M-theory permit dS vacua. A partial answer 
to this question is provided by a no-go theorem of Gibbons [4] which states that the $D$ dimensional Einstein field equations do not permit a time-independent compactification to a $\mathrm{dS}$ spacetime of dimension $d<D$ if the stress tensor satisfies the Strong Energy Condition (SEC); this follows from a simple inequality implied by the higher-dimensional SEC. Although the SEC is not as fundamental as the Dominant Energy Condition (DEC), it is satisfied (with one exception to be mentioned below) by $\mathrm{D}=10 / 11$ supergravity theories, so this rules out time-independent compactifications to $\mathrm{dS}$ of these theories. This no-go theorem was rediscovered in a string/M-theory context by Maldacena and Nuñez [5], who extended the result to the massive IIA $D=10$ supergravity; this has a dilaton potential that violates the SEC, but a lower-dimensional dS universe is still unobtainable by a timeindependent compactification.

In principle, this Gibbons-Maldacena-Nuñez (GMN) no-go theorem might be circumvented by relaxing the usual non-singularity condition on the compact spaces used for compactification, since some geometrical singularities are innocuous in String/M-theory. An influential model of this type (incorporating $D$-branes and, crucially, orientifold planes) has been argued to yield a dS vacuum [6] (see [7] for a recent overview). However, despite more than a decade of active investigation on this topic it was possible to ask less than one year ago "What if string theory has no de Sitter vacua?" [8]. More recently, it has been suggested that dS vacua belong to the string theory "swampland" [9].

Another potential way around the GMN no-go theorem is to relax the condition of time-independence. If this is done then it is important to distinguish between $d$-metrics in different conformal frames; these differ by a scalar-field dependent conformal factor which may become time-dependent for time-dependent compactifications. It is usual to insist on Einstein conformal frame because the $d$-dimensional Newton constant is otherwise timedependent, and this would be in conflict with observations for $d=4$. The first attempt to generalize the GMN theorem to allow for a time-dependent compact space yielded the conclusion that the SEC still prevents compactification to dS [10], and some analogous results were presented more recently in [9]. This conclusion, however, is based on some unnecessary assumptions that we make explicit here.

Our conclusion is not that compactification to $\mathrm{dS}$ is still excluded when time-dependence is allowed, but rather that any example of it must implement the Einstein-frame condition in a way that differs from the way it has been implemented previously, at least in those time-dependendent cosmological compactifications of which we are aware. It is not clear to us how this loophole can be exploited in practice, so we focus here on a different question: assuming that the Einstein-frame condition is implemented in the customary way, and that compactification to $\mathrm{dS}$ is then excluded (as we confirm) is late-time cosmic acceleration also excluded?

It is known that the higher-dimensional SEC does not prevent compactification to an Einstein-frame FLRW universe that undergoes accelerated expansion for some finite period [11. For a $D$-dimensional stress tensor satisfying the SEC, many other time-dependent compactifications have been found that lead to a similar transient cosmic acceleration of the lower-dimensional Einstein-frame metric [12, 13, 14, 15, 16, 17] but none with late-time acceleration. Indeed, this appears to be ruled out by Teo's generalized no-go theorem [10] 
but the proof implicitly uses the vacuum Einstein equations in the higher dimension. This leaves open the possibility that some compactification to an FLRW universe undergoing late-time acceleration might be achieved by a non-zero stress tensor satisfying the SEC in the higher dimension.

An FLRW cosmology that undergoes late-time accelerated expansion can be expected to approach a scaling solution of the Einstein equations for which the accelerated expansion is eternal because these solutions are late-time attractors (in contrast to non-accelerating scaling solutions). If the SEC were to forbid compactification to eternally accelerating universes then this would constitute strong evidence against compactification to any universe that undergoes late-time acceleration (i.e. with an expansion rate that is strictly increasing from some time $\left.t_{0}\right)$. However, what we find is that the SEC does not forbid compactification to eternally accelerating universes. Specifically, we exhibit cosmological compactifications of the $D$-dimensional Einstein equations, with a stress tensor satisfying both the SEC and the DEC, such that the lower dimensional FLRW universe undergoes a power-law accelerated expansion. A field theory realization of the $D$-dimensional stress tensor, which could allow the exploration of more general solutions, remains an open problem, as does any connection to string/M-theory.

An interesting feature of these cosmological compactifications is that the compact space is also expanding, which implies an ultimate decompactification. However, it is remarkable that an accelerating expansion of the FLRW universe requires a decelerating expansion of the compact space. We explore the implications for our Universe on the assumption that it arises from some compactification of this type. Our conclusion is that the decompactification time (which we define as the time at which effects of Kaluza-Klein particles would show up in current accelerator experiments) is arbitrarily long, and easily much longer than the current age of the universe.

From a $d$-dimensional perspective, the conclusion of the GMN no-go theorem can be restated as the condition that the scalar potential $V$ of the effective $d$-dimensional theory cannot have stationary points with $V=V_{0}>0$ [18]. The occurrence of transient cosmic acceleration is then simply explained in terms of the properties of such potentials [19]: given a potential that is positive in some region of field space, there exist initial conditions such that this region is entered, at which point the motion in field space is 'uphill'. If there are no stationary points in the region of positive potential then this uphill motion must end, at which moment the FLRW universe will be accelerating. How long it continues to accelerate depends on details of the potential, in particular its gradient $\nabla V$. Late-time acceleration will occur only if the path in field space is such that $V$ remains positive and the late-time value of $|\nabla V| / V$ is subcritical.

In our examples of late-time accelerated expansion, the $d$-dimensional FLRW universe is filled with a perfect fluid with a constant pressure/energy-density ratio $w$, but this constant is restricted to lie in a particular interval. In the four-dimensional case,

$$
-\frac{1}{2}<w<-\frac{1}{3} \quad(d=4) .
$$

The upper bound on $w$ is simply a consequence of our assumption of accelerated expansion; 
the lower bound is implied by the higher-dimensional SEC. Since $w=-1$ corresponds to a $\mathrm{dS}$ universe, this result constitutes a significant generalization of the GMN no-go theorem to the time-dependent case, on assumptions that are the same as (or weaker than) those used previously (e.g. [9, 10]) to rule out time-dependent compactifications to dS. Of course, the restrictions on $w$ apply only at late-times; current observations are compatible with $w=-1$ but they are also compatible with a time-dependent increasing $w$ [20].

A feature of FLRW universes undergoing power-law expansion is that they can also be realized as solutions of the $d$-dimensional Einstein equations for gravity coupled to a scalar field $\phi$ with positive 'exponential' potential $V \propto e^{\lambda \phi}$, for constant $\lambda=V^{\prime} / V$ [21] (see [18] for the $d$-dimensional generalization). In fact, the general FLRW solution of such a model can be found exactly [22]; it approaches a power-law solution at early and late times, with late-time acceleration only if $\lambda<\lambda_{c}$, where $\lambda_{c}$ is a 'critical' value of order unity (its precise value is both $d$-dependent and convention dependent).

Scalar-tensor theories of this type typically arise as consistent truncations of compactified $\mathrm{D}=10 / 11$ supergravity theories and it was conjectured in [23] (based on a variety of examples) that this always leads to $\lambda>\lambda_{c}$; this conjecture was proved in [10] but subject to a restrictive implicit assumption (that we make explicit here). A subsequent study for generic multi-scalar theories with positive potential $V(\phi)$ focused on the time evolution of the vector of "characteristic functions" $\partial \ln V / \partial \phi$ [24]. These results are presumably related to the more recent conjecture that positive potentials arising from string/M-theory compactifications are such that $|\nabla V| / V \geq c$ for some constant $c[9]$; we comment on this proposal in our conclusions.

The organization is as follows. We first derive the general SEC inequality for timedependent compactifications to an FLRW universe. This is a new result since previous SEC inequalities have all made assumptions that are not required by FLRW isometries. We discuss the difficulty of drawing definite conclusions from this inequality, and then proceed to make the simplifying assumption concerning implementation of the Einstein-frame condition that is implicit in previous investigations of time-dependent compactifications. This takes us to a simpler SEC inequality; we analyse its implications, confirming that it excludes compactification to dS. We then analyse the implications for compactifications to FLRW universes with power-law expansion, finding examples for which the expansion is accelerating. In section 3 we turn to an investigation of these examples, examining the implications for decompactification, showing that the required stress tensor in $D$-dimensions satisfies the DEC in addition to the SEC, and finding the restriction (1.1) on the lowerdimensional stress tensor. As the compact space in our examples is a scale factor times a Ricci-flat metric, we briefly consider the implications of relaxing the Ricci flat condition. We conclude with a summary of our main results and a further discussion of constraints on scalar potentials in the $d$-dimensional effective scalar-tensor gravity theory. 


\section{Warped compactifications to FLRW cosmologies}

The general $d$-dimensional FLRW spacetime has a metric of the form

$$
d s_{F L R W}^{2} \equiv g_{\mu \nu} d x^{\mu} d x^{\nu}=-d t^{2}+S^{2}(t) \bar{g}_{i j} d x^{i} d x^{j},
$$

where $S(t)$ is the scale factor as a function of standard FLRW time, and $\bar{g}_{i j}$ is the metric in local coordinates $\left\{x^{i} ; i=1, \cdots, d-1\right\}$ for a maximally-symmetric $(d-1)$-space with constant curvature $k$, which we leave un-normalized. Our starting point will be a $D$-dimensional manifold that is topologically a product of this FLRW spacetime with a compact $n$-dimensional manifold $B$ (so $D=d+n$ ). The most general $D$-metric compatible with the FLRW isometries, which are those of $\bar{g}_{i j}$, has the form

$$
d s_{D}^{2}=\Omega^{2}(y ; t) d s_{F L R W}^{2}+h_{\alpha \beta}(y ; t) d y^{\alpha} d y^{\beta},
$$

where $h_{\alpha \beta}$ is the metric on $B$ in local coordinates $\left\{y^{\alpha} ; \alpha=1, \ldots, n\right\}$, and $\Omega$ is a nowherezero 'warp factor': a scalar function on $B$. Notice that the FLRW isometries permit not only the metric on the compact manifold $B$ to be time-dependent but also the warp factor.

The condition for $d s_{F L R W}^{2}$ to be an Einstein frame metric for the effective $d$-dimensional gravity theory is

$$
\int_{B} d^{n} y \sqrt{\operatorname{det} h} \Omega^{d-2}=G_{D} / G_{d}
$$

where the constant on the right-hand side is the ratio of the Newton constants in the higher and lower spacetime dimensions. This motivates the following notation: for any scalar $\Phi$ on $B$, we shall define

$$
\langle\Phi\rangle=\left(G_{d} / G_{D}\right) \int_{B} d^{n} y \sqrt{\operatorname{det} h} \Omega^{d-2} \Phi .
$$

The Einstein-frame condition in this notation is $\langle 1\rangle=1$, which means that $\langle\Phi\rangle$ can be interpreted as an average of $\Phi$ over $B$; this means, in particular, that $\langle\Phi\rangle=\Phi$ when $\Phi$ is independent of position on $B$.

As the Einstein-frame condition must hold for all $t$ we may deduce, by taking its time derivative, the following "first-order" Einstein-frame condition:

$$
\frac{1}{2}\left\langle\operatorname{tr}\left(h^{-1} \dot{h}\right)\right\rangle=-(d-2)\langle\dot{\Omega} / \Omega\rangle .
$$

By taking another derivative we deduce the "second-order" Einstein-frame condition

$$
\frac{1}{2}\left\langle\operatorname{tr}\left(h^{-1} \ddot{h}\right)\right\rangle=\frac{1}{2}\left\langle\operatorname{tr}\left(h^{-1} \dot{h}\right)^{2}\right\rangle+(d-2)\left\langle\left[(\dot{\Omega} / \Omega)^{2}-(\ddot{\Omega} / \Omega)\right]\right\rangle-\left\langle X^{2}\right\rangle,
$$

where

$$
X \equiv \frac{1}{2} \operatorname{tr}\left(h^{-1} \dot{h}\right)+(d-2) \dot{\Omega} / \Omega .
$$

Notice that $X$ averages to zero as a consequence of 2.5 ; however, this does not imply that the same is true of $X^{2}$. 


\subsection{The Strong Energy Condition}

The SEC on the stress tensor in $D$ dimensions implies, via the Einstein field equations, that the time-time component of the Ricci tensor is non-negative. A direct calculation of this Ricci tensor component yields

$$
\begin{aligned}
R_{00}= & -\frac{1}{2} \operatorname{tr}\left(h^{-1} \ddot{h}\right)-(d-1)\left(\frac{\ddot{S}}{S}+\frac{\ddot{\Omega}}{\Omega}\right)+\frac{1}{d} \Omega^{2-d} \nabla^{2} \Omega^{d} \\
& +(d-1)\left[(\dot{\Omega} / \Omega)^{2}-(\dot{\Omega} / \Omega)(\dot{S} / S)\right]+\frac{1}{2}\left(\frac{\dot{\Omega}}{\Omega}\right) \operatorname{tr}\left(h^{-1} \dot{h}\right)+\frac{1}{4} \operatorname{tr}\left[\left(h^{-1} \dot{h}\right)^{2}\right] .
\end{aligned}
$$

If we average over $B$ then we may use the second-order Einstein-frame condition (2.6), together with

$$
\left\langle\Omega^{2-d} \nabla^{2} \Omega^{d}\right\rangle=: \int_{B} d^{n} y \sqrt{\operatorname{det} h} \nabla^{2} \Omega=\int_{B} d^{n} y \nabla_{\alpha}\left(\sqrt{\operatorname{det} h} h^{\alpha \beta} \partial_{\beta} \Omega^{d}\right)=0,
$$

to deduce that

$$
\begin{aligned}
\left\langle R_{00}\right\rangle= & -(d-1)[(\ddot{S} / S)+(\dot{S} / S)\langle\dot{\Omega} / \Omega\rangle]-\langle\ddot{\Omega} / \Omega\rangle-(d-3)\left\langle(\dot{\Omega} / \Omega)^{2}\right\rangle \\
& -\frac{1}{4}\left\langle\operatorname{tr}\left(h^{-1} \dot{h}\right)^{2}\right\rangle+\langle X[X+\dot{\Omega} / \Omega]\rangle .
\end{aligned}
$$

The left hand side is non negative if the SEC is satisfied, so a necessary condition for the SEC to hold is that the right hand side is non-negative. This inequality is

$$
\begin{aligned}
& -(d-1)[(\ddot{S} / S)+(\dot{S} / S)\langle\dot{\Omega} / \Omega\rangle]-\langle\ddot{\Omega} / \Omega\rangle-(d-3)\left\langle(\dot{\Omega} / \Omega)^{2}\right\rangle+\langle X[X+\dot{\Omega} / \Omega]\rangle \\
& \geq \frac{1}{4}\left\langle\operatorname{tr}\left(h^{-1} \dot{h}\right)^{2}\right\rangle \geq \frac{1}{4 n}\left\langle\left[\operatorname{tr}\left(h^{-1} \dot{h}\right)\right]^{2}\right\rangle
\end{aligned}
$$

where the second inequality, which is saturated when the $n \times n$ matrix $h^{-1} \dot{h}$ is proportional to the identity matrix, follows from the fact that $\operatorname{tr}\left[M-n^{-1} \operatorname{tr} M\right]^{2} \geq 0$ for any $n \times n$ matrix $M$; the resulting inequality is again a necessary condition for the SEC. This last step allows us to rewrite the right hand side of (2.11) using the identity

$$
\left[\frac{1}{2} \operatorname{tr}\left(h^{-1} \dot{h}\right)\right]^{2} \equiv(d-2)^{2}(\dot{\Omega} / \Omega)^{2}+X^{2}-2(d-2) X(\dot{\Omega} / \Omega) .
$$

This yields the following integrated SEC inequality

$$
\begin{aligned}
& -(d-1)[(\ddot{S} / S)+(\dot{S} / S)\langle\dot{\Omega} / \Omega\rangle]-\langle\ddot{\Omega} / \Omega\rangle+\left(1-a^{-2}\right)\left\langle(\dot{\Omega} / \Omega)^{2}\right\rangle \\
& +\frac{(n-1)}{n}\left\langle X^{2}\right\rangle+\left(1+\frac{2(d-2)}{n}\right)\langle X(\dot{\Omega} / \Omega)\rangle \geq 0,
\end{aligned}
$$


where we have introduced the constant

$$
a=\sqrt{\frac{n}{(D-2)(d-2)}} .
$$

This inequality is saturated when the $D$-dimensional averaged SEC is saturated and when only the size of the compact space $B$ (and not its shape) is time-dependent.

If there is a time $t_{0}$ for which all first derivatives are zero then the integrated SEC inequality 2.13) implies that, at this time,

$$
-(d-1)(\ddot{S} / S)-\langle\ddot{\Omega} / \Omega\rangle \geq 0 \quad\left(t=t_{0}\right),
$$

which is the inequality found in [9]. It does not exclude acceleration ${ }^{1}$ because a negative contribution to the left hand side from a positive $\ddot{S}$ can be overcome by a positive contribution from a negative $\ddot{\Omega}$. Indeed, this is precisely the mechanism for the transient acceleration found in the time-dependent compactifications mentioned in the Introduction: the acceleration occurs as the volume of the compact space passes through a minimum value, corresponding to a maximum of $\Omega(t)$.

The issue that we wish to address is whether the SEC permits late-time acceleration. At late-times we expect some scaling solution of the Einstein equations for which $\dot{\Omega}$ and $\dot{S}$ are, generically, never zero, in which case it is (2.13), rather than (2.15), that has implications for late-time behaviour.

\section{$2.2 \quad$ No-go theorems}

If $\dot{\Omega} \equiv 0$ then 2.13 reduces to

$$
\ddot{S} \leq 0
$$

which tells us that the expansion of the universe is non-accelerating. This result incorporates the GMN theorem but is more general for two reasons. Firstly, it applies to any FLRW spacetime, not just dS. Secondly, $\dot{\Omega} \equiv 0$ does not imply time-independence of the metric on $B$; it implies only (via the Einstein-frame condition) that the integral $\int_{B} \Omega^{d-2} \operatorname{vol}_{B}$ is constant (where $\operatorname{vol}_{B}$ is the volume $n$-form associated to the metric $h$ ).

If $\dot{\Omega} \not \equiv 0$ then there is no obvious conclusion to be drawn from the inequality $(2.13)$, principally because the final term on the left hand side could be positive or negative. It could also be zero, and is zero if the Einstein-frame condition is realized in the form

$$
\sqrt{\operatorname{det} h} \Omega^{d-2}=\omega(y), \quad \int_{B} d^{n} y \omega(y)=G_{D} / G_{d} .
$$

\footnotetext{
${ }^{1}$ Here we appear to have a disagreement with Section 2.4 of [9], not only because (2.15) does not rule out transient acceleration (as explained in what follows), but also because the acceleration bound (2.12) of [9] is derived for a late-time (power-law) attractor solution for which (2.15) does not apply because first derivatives are not zero.
} 
In this case the first-order Einstein frame condition (2.5) simplifies to

$$
\frac{1}{2} \operatorname{tr}\left(h^{-1} \dot{h}\right) \equiv-(d-2)(\dot{\Omega} / \Omega) \quad(\Leftrightarrow X \equiv 0) .
$$

The inequality (2.13) then reduces to ${ }^{2}$

$$
-(d-1)[(\ddot{S} / S)+(\dot{S} / S)\langle\dot{\Omega} / \Omega\rangle]-\langle\ddot{\Omega} / \Omega\rangle+\left(1-a^{-2}\right)\left\langle(\dot{\Omega} / \Omega)^{2}\right\rangle \geq 0 .
$$

This generalizes the bound derived in [9] to the case where $\dot{\Omega}$ and $\dot{S}$ are different from zero. They are never zero for the accelerating scaling solutions that we shall exhibit later.

As before, the inequality (2.19) is saturated when the $D$-dimensional averaged SEC is saturated and only the size of the compact space $B$ is time-dependent. It excludes a latetime dS universe, i.e. one for which $S(t) \sim e^{H t}$ for positive constant $H$. To see this, observe that the $\ddot{S} / S$ term will dominate (leading to a contradiction) unless $\Omega$ also increases faster than any polynomial, and if this increase is faster than exponential then the $\ddot{\Omega} / \Omega$ term will dominate (again leading to a contradiction), so we must suppose that $\Omega(y ; t)=\tilde{\Omega}(y) e^{J t}$ for constant $J$. The inequality (2.19) then yields the following quadratic inequality in $(H, J)$ :

$$
H^{2}+H J+\frac{(D-2)(d-2)}{n(d-1)} J^{2} \leq 0 .
$$

This equation has no real solutions, so compactification to dS is excluded.

This does not prove that the SEC forbids a time-dependent compactification to dS because the Einstein-frame condition (2.3) could be satisfied even though its unaveraged form (2.17) is not, and in this case we would have to return to the inequality 2.13). Nevertheless, we think it worthwhile to explore the implications of the SEC given the assumption that the unaveraged Einstein-frame condition is satisfied. As far as we are aware, this unaveraged form has been assumed in all previous work on time-dependent cosmological compactifications.

\subsection{Power-law acceleration}

Having confirmed that compactification to dS is excluded under the assumption that the Einstein-frame condition is satisfied in its unaveraged form (2.17), we now investigate whether this assumption allows a time-dependent compactification to an FLRW universes undergoing late-time acceleration; i.e. we assume that $S \sim t^{\eta}$ at late time, for some constant $\eta$, and seek scaling solutions of this type. In this case, the inequality $(2.19)$ does not allow exponential growth of $\Omega$, so we shall suppose that $\Omega \sim t^{\xi}$ at late time, for some other constant $\xi$. The asymptotic solution must then take the form ${ }^{3}$

$$
S=t^{\eta}, \quad \Omega=\Omega_{0}(y) t^{\xi},
$$

\footnotetext{
${ }^{2}$ This is equivalent to the inequality found in [10] for $d=4$, but the subsequent analysis there limits the results to vacuum solutions only.

${ }^{3}$ Strictly speaking, the SEC also allows $S=t^{\eta} f_{1}(t)$, and $\Omega=\Omega_{0} t^{\xi} f_{2}(t)$ if $t f^{\prime} / f \rightarrow 0$ and $t^{2} f^{\prime \prime} / f \rightarrow 0$ as $t \rightarrow \infty$, but this still leads to the inequality 2.22 .
} 
where a normalization constant for $S(t)$ is omitted because it can be absorbed into $\bar{g}_{i j}$. The inequality 2.19) now becomes

$$
\eta^{2}+\eta \xi+\frac{(D-2)(d-2)}{n(d-1)} \xi^{2}-\eta-\frac{1}{(d-1)} \xi \leq 0 .
$$

This defines the interior of an ellipse in the $(\eta, \xi)$ plane. For an accelerating universe we must also have $\eta>1$, but the line $\eta=1$ intersects the ellipse so there is a region inside the ellipse for which $\eta>1$. The largest value of $\eta$ allowed by the SEC corresponds to the line $\eta=\eta_{\max }$ that is tangent to the ellipse, which occurs when

$$
\eta_{\max }=\frac{(d-1)\left(2-a^{2}\right)+2 \sqrt{(d-1)^{2}-a^{2}(d-1)(d-2)}}{(d-1)\left(4-a^{2}(d-1)\right)}<\frac{4}{3},
$$

where the upper bound is approached as both $d \rightarrow \infty$ and $n \rightarrow \infty$; in contrast $\eta_{\max } \rightarrow 1$ as as $d \rightarrow \infty$ for fixed $n$. Within the region $1 \leq \eta \leq \eta_{\max }$ the parameter $\xi$ is bounded by

$$
-\frac{n}{D-2} \leq \xi \leq 0
$$

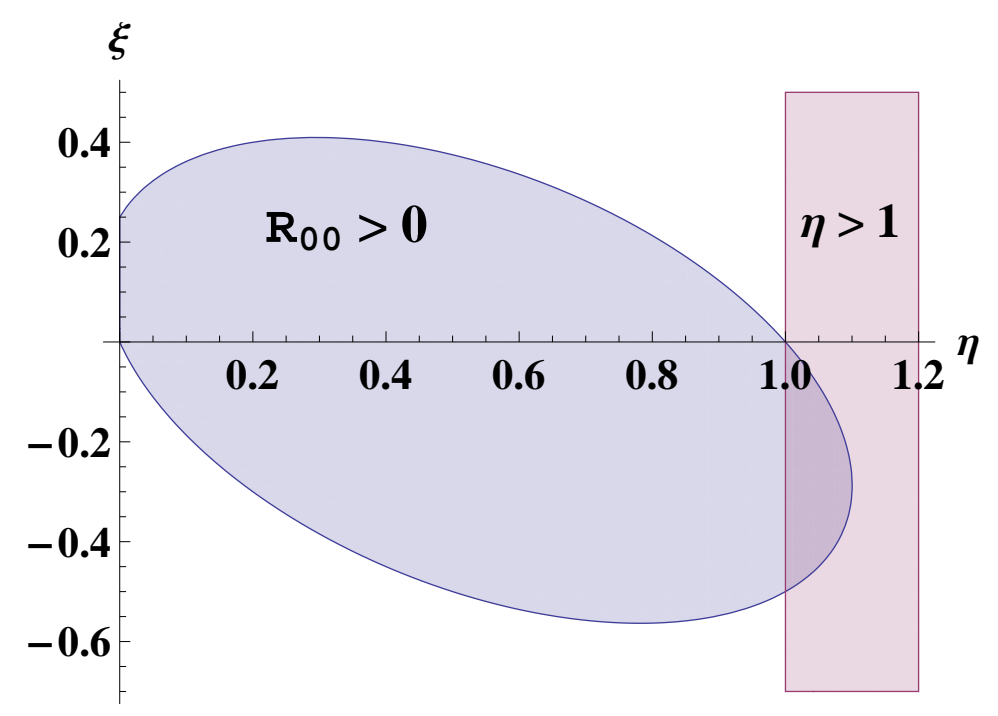

Figure 1: The SEC $R_{00} \geq 0$ is the region inside the solid ellipse (here $D=6, d=4$ ). The part of the region $\eta>1$ inside this ellipse describes accelerating universes satisfying SEC (this region is inside the much larger region allowed by DEC, which we determine in section 3.2 ).

The restriction to negative $\xi$ tells us that the compact space is expanding This implies an eventual decompactification, which we now investigate.

\footnotetext{
${ }^{4}$ If the bound (2.15) derived in [9] were to be used then one would conclude (incorrectly) that $\xi$ is positive, and hence that the compact space is contracting, but this bound does not apply here since neither $\dot{S}$ nor $\dot{\Omega}$ is zero at late times.
} 


\subsection{Decompactification}

Let $\varphi(t)$ be the scale factor for the $n$-dimensional compact space; i.e.

$$
\varphi^{n} \propto \sqrt{\operatorname{det} h} .
$$

Since

$$
\frac{d}{d t} \sqrt{\operatorname{det} h}=-(d-2)(\dot{\Omega} / \Omega) \sqrt{\operatorname{det} h}=-\frac{(d-2) \xi}{t} \sqrt{\operatorname{det} h},
$$

we have

$$
\dot{\varphi}=-\frac{(d-2) \xi}{n t} \varphi,
$$

which has the solution

$$
\varphi \propto t^{\sigma}, \quad \sigma=-\frac{(d-2) \xi}{n} .
$$

When expressed in terms of $\sigma$ the restriction 2.24 ) on $\xi$ implies (for $\xi \neq 0$ ) that

$$
0<\sigma \leq \frac{d-2}{D-2} .
$$

This confirms that the compact space is expanding (as $\sigma>0$ ) and it additionally tells us that this expansion is decelerating (as $\sigma<1$ ).

A feature of additional compact dimensions is that the harmonic expansion of fields on these dimensions will lead to an infinite sequence of Kaluza-Klein (KK) particles with some lowest mass set by the scale of the compact space. Let $M_{0}$ be the mass of the lightest KK particle at time $t_{0}$; then the corresponding mass at a later time $t$ will be

$$
M(t)=\left(t_{0} / t\right)^{\sigma-\xi} M_{0} \quad\left(t>t_{0}\right) .
$$

This formula takes into account the the time-dependence arising from the warp factor $\Omega$ in addition to the the time-dependence of the volume of the compact space metric coming from its scale factor $\varphi$. From the formula for $\sigma$ in (2.28) we see that

$$
\sigma-\xi=-\frac{(D-2)}{n} \xi
$$

and hence, since $\xi \leq 0$,

$$
M(t)=\left(t_{0} / t\right)^{(D-2)|\xi| / n} M_{0} .
$$

This is a decreasing function of time, and the existence of the KK tower of particles will have observable consequences at some "decompactification time" $T>t_{0}$ when $M(T)$ is of the order of particle masses accessible to particle accelerators. The relation between $T$ and $M(T)$ is

$$
\frac{M_{0}}{M(T)}=\left(\frac{T}{t_{0}}\right)^{\frac{(D-2)|\xi|}{n}}
$$


but we also have $S(T)=\left(T / t_{0}\right)^{\eta}$, and hence

$$
S(T)=\left(\frac{M_{0}}{M(T)}\right)^{\gamma}, \quad \gamma=\frac{n \eta}{(D-2)|\xi|} \geq 1,
$$

where the lower bound on $\gamma$ is a consequence of the bound (3.16) on $\xi$ and the assumption that $\eta>1$. This tells us how much the FLRW universe has expanded prior to its effective decompactification to a $D$-dimensional universe. Of course, this is an approximation because we are using only the late-time solution.

To get an idea for the likely numbers in any attempt to apply these ideas to a realistic model, let us take $M_{0}$ to be the Planck mass and $M(T)$ the mass of the Higgs boson. Then $M_{0} / M(T) \sim 10^{17}$, so

$$
S(T) \sim 10^{17 \gamma}
$$

This is arbitrarily large because there is no upper bound on $\gamma$; this means that $T$ could be much longer than the current age of the universe. However, one might suspect that fine tuning is needed for $\gamma \gg 1$, so it is of interest to ask how large $\gamma$ must be to avoid a conflict with the current lack of evidence for extra dimensions. If $t_{0}$ is the time at the end of inflation (which is the earliest time that we could reasonably choose) then we would need $S(T) \gg 10^{30}$ to be sure of consistency with the current non-appearance of KK particles. This imposes a phenomenological bound that is approximately $\gamma>2$.

\subsection{SEC Redux}

Now that we have found a possibility for compactification to an accelerating FLRW universe, we must recall that the integrated SEC inequality is merely a necessary condition for validity of the SEC. This means that we must return to the unintegrated condition $R_{00} \geq 0$ and ask whether this is also satisfied. As we are now assuming that the Einstein-frame condition is satisfied in unintegrated form, we may use this to simplify the expression (2.8) for $R_{00}$, and hence the unintegrated SEC $R_{00} \geq 0$; one finds that

$$
\begin{aligned}
& -(d-1)[(\ddot{S} / S)+(\dot{S} / S)(\dot{\Omega} / \Omega)]-(\ddot{\Omega} / \Omega)+\left(1-a^{-2}\right)(\dot{\Omega} / \Omega)^{2} \\
& +\frac{1}{d} \Omega^{2-d} \nabla^{2} \Omega^{d} \geq 0 .
\end{aligned}
$$

This reduces to 2.19) upon taking the average over $B$, but the unaveraged inequality involves an additional term proportional to $\Omega^{2-d} \nabla^{2} \Omega^{d}$. Since this term averages to zero it must be negative somewhere on the compact space $B$ unless it is identically zero on $B$, which would require $\Omega$ to be (time-dependent) constant on $B$.

What this means is that compactification metrics with $\Omega$ of the form assumed above in (2.21) are not guaranteed to satisfy the SEC inequality, even if they do satisfy the integrated SEC inequality, unless we further assume that $\Omega_{0}$ is constant on $B$. It may be that for some non-constant choices of $\Omega_{0}$ the unintegrated SEC inequality (2.36) will be satisfied, but more work is required to answer this question. For this reason, we shall assume in the following section that $\Omega_{0}$ is a constant. 


\section{The late-time stress tensor}

We have now seen that the SEC permits compactification to eternally accelerating universes for which

$$
S(t)=t^{\eta}, \quad \Omega(t)=\Omega_{0} t^{\xi},
$$

but we do not yet know anything about the stress tensor that makes this possible. For example, we do not yet know whether it could be zero, although we shall soon see that this is not a possibility.

A calculation, using (3.1) and (2.18), yields

$$
\begin{aligned}
& R_{00}=-\frac{(d-1)}{t^{2}}\left\{\eta^{2}+\eta \xi+\frac{(D-2)(d-2)}{n(d-1)} \xi^{2}-\eta-\frac{1}{(d-1)} \xi\right\}, \\
& R_{i j}=\left\{t^{2(\eta-1)}(\xi+\eta)[(d-1) \eta-1]+(d-2) k\right\} \bar{g}_{i j} .
\end{aligned}
$$

The space-curvature term proportional to $k$ in the expression for $R_{i j}$ is non-leading as $t \rightarrow \infty$ when $\eta>1$, which is precisely the condition for accelerated expansion. For this reason we ignore this term in what follows, although we will re-instate it later when we consider the $\eta=1$ case, since zero acceleration for $t=\infty$ does not exclude a positive acceleration for large but finite $t$.

We shall further suppose here that only the volume of $B$ in the metric $h$ is timedependent; i.e.

$$
h_{\alpha \beta}=\varphi^{2}(t) \tilde{h}_{\alpha \beta}(y),
$$

where $\varphi$ is a scale factor for the compact space $B$. In this case,

$$
\operatorname{tr}\left(h^{-1} \dot{h}\right)=2 n(\dot{\varphi} / \varphi), \quad \operatorname{tr}\left(h^{-1} \dot{h}\right)^{2}=4 n(\dot{\varphi} / \varphi)^{2} .
$$

Using (2.18) again we learn that

$$
\varphi \propto t^{-(d-2) \xi / n} .
$$

In addition, we shall suppose that the metric $h$ on $B$ is Ricci flat; we return later to consider the implications of allowing it to be a generic Einstein metric on $B$. Under this Ricci-flat assumption, a further calculation shows that

$$
R_{\alpha \beta}=-t^{-2(1+\xi)}[(d-2) / n][(d-1) \eta-1] \xi h_{\alpha \beta},
$$

from which we deduce the following expression for the Ricci scalar:

$$
R=t^{-2(1+\xi)}\left\{d(d-1) \eta^{2}+2(d-1) \eta \xi+\frac{(d-2)(D-2)}{n} \xi^{2}-2(d-1) \eta-2 \xi\right\} .
$$

All other components of the Ricci tensor are zero.

From these results we find that the non-zero components of the Einstein tensor take the form 5

$$
G_{00}^{(D)}=\frac{A}{t^{2}}, \quad G_{i j}^{(D)}=\frac{B}{t^{2}} g_{i j}, \quad G_{\alpha \beta}^{(D)}=\frac{C}{t^{2(1+\xi)}} h_{\alpha \beta},
$$

\footnotetext{
${ }^{5}$ We recall that $g_{i j}=S^{2} \bar{g}_{i j}$.
} 
where the three constants $(A, B, C)$ are (after setting $k=0$ for the reasons explained above)

$$
\begin{aligned}
A= & \frac{(d-2)}{2}\left[(d-1) \eta^{2}-\frac{(D-2)}{n} \xi^{2}\right], \\
B= & \frac{(d-2)}{2}\left[-(d-1) \eta^{2}-\frac{(D-2)}{n} \xi^{2}+2 \eta\right], \\
C= & -\frac{d(d-1)}{2} \eta^{2}-\frac{(d-1)(D-2)}{n} \eta \xi-\frac{(d-2)(D-2)}{2 n} \xi^{2} \\
& +(d-1) \eta+\frac{(D-2)}{n} \xi .
\end{aligned}
$$

The contracted Bianchi identities satisfied by the Einstein tensor are equivalent to the identity

$$
[2+\xi-(d-1) \eta] A-(d-1)(\eta+\xi) B+(d-2) \xi C \equiv 0
$$

as substitution for $(A, B, C)$ in terms of $(\eta, \xi)$ confirms.

The vacuum Einstein equations in $D$-dimensions now reduce to $A=B=C=0$, but elimination of $\xi$ from the two equations $A=B=0$ yields the equation $\eta[(d-1) \eta-1]=0$, which implies that $\eta<1$; this agrees with the result of [10], where the vacuum equations were used to simplify the SEC inequality.

We now know that the stress tensor required for $\eta>1$ must be non-zero. We shall take its non-zero components to be

$$
T_{00}=t^{-2} \rho_{0}, \quad T_{i j}=t^{-2} P_{0} g_{i j}, \quad T_{\alpha \beta}=t^{-2(1+\xi)} P_{0}^{(\mathrm{int})} h_{\alpha \beta},
$$

for constants $\left\{\rho_{0}, P_{0}, P_{0}^{(\text {int })}\right\}$. This choice is consistent with the Bianchi identities provided that

$$
[2+\xi-(d-1) \eta] \rho_{0}-(d-1)(\eta+\xi) P_{0}+(d-2) \xi P_{0}^{(\mathrm{int})}=0 .
$$

For a convenient choice of units, the late time Einstein equations are now

$$
A=\rho_{0} \quad B=P_{0}, \quad C=P_{0}^{(\text {int })},
$$

but only two of these three equations are independent because of the linear relations 3.10 and (3.12). We may take the first and third equations as the independent ones if $\xi=0$, but it is then straightforward to show that the SEC cannot be satisfied for $\eta>1$; this is expected because $\xi=0$ implies a time-independent metric for the compact manifold $B$. We may therefore assume that $\xi \neq 0$, and in this case we may take the first two of the equations 3.9 as the two independent equations; these are

$$
\begin{aligned}
& \rho_{0}=\frac{(d-2)}{2}\left[(d-1) \eta^{2}-\frac{(D-2)}{n} \xi^{2}\right] \\
& P_{0}=-\frac{(d-2)}{2}\left[(d-1) \eta^{2}-2 \eta+\frac{(D-2)}{n} \xi^{2}\right] .
\end{aligned}
$$


Using $\eta>1$ and $|\xi|<n /(D-2)$, from $(2.24)$, one may easily show that

$$
\rho_{0}>0, \quad P_{0}<0 .
$$

The pressure constant $P_{0}^{(\mathrm{int})}$ is determined by the linear relation 3.12 (or, equivalently, by the third Einstein equation):

$$
P_{0}^{(\mathrm{int})}=-\frac{(d-1)}{2}\left[d \eta^{2}-2 \eta\right]-\frac{(D-2)}{n} \xi\left[(d-1) \eta-1+\frac{(d-2)}{2} \xi\right] .
$$

Using again the restriction 2.24 ) on the range of $\xi$ allowed by the SEC, and the assumption that $\eta>1$, we have

$$
P_{0}^{(\mathrm{int})}<-\frac{(d-2)(d-3)}{2}<0 .
$$

The stress tensor defined by (3.11) suggests some anisotropic generalization of a perfect fluid (possibly along the lines of of [25]) with the time-dependence determined in terms of the two scale factors $(S, \varphi)$ by some analog of the continuity equation. If so, one might expect some field theoretic realization, possibly with the fields of $D=10 / 11$ supergravity. This is an interesting open problem but the effective $d$-dimensional stress tensor is a perfect fluid with a scalar field theoretic realization, as we now discuss.

\subsection{A new no-go theorem}

We have considered a class of cosmological compactification solutions of the $D$-dimensional Einstein field equations for which the Einstein-frame metric in the lower dimension is an FLRW spacetime with scale factor $S^{\eta}$, and we have shown that the SEC in $D$ dimensions is compatible with $\eta>1$, i.e. accelerated expansion. The Einstein tensor for this FLRW metric has non-zero components

$$
G_{00}=\frac{A_{0}}{t^{2}} \equiv \rho, \quad G_{i j}=\frac{B_{0}}{t^{2}} g_{i j} \equiv P g_{i j}
$$

where

$$
A_{0}=\frac{(d-2)}{2}\left[(d-1) \eta^{2}\right], \quad B_{0}=-\frac{(d-2)}{2}\left[(d-1) \eta^{2}-2 \eta\right] .
$$

These are the coefficients $A$ and $B$ of $(3.9)$ at $\xi=0$, which are those required for consistency with the Bianchi identity satisfied by the Einstein tensor for the Einstein-frame $d$-metric.

The $d$-dimensional stress tensor is that of a perfect fluid with mass density $\rho$ and pressure $P$ satisfying the continuity equation

$$
\dot{\rho}=-(d-1)(\rho+P)(\dot{S} / S),
$$

as one may verify. The equation of state is

$$
P=w \rho, \quad w=B_{0} / A_{0}=-1+\frac{2}{(d-1) \eta} .
$$


This equation of state is what one finds, under the assumptions of homogeneity and isotropy, for a scalar field in $d$-dimensions with a positive exponential potential, which has a subcritical exponent when $\eta>1$. The only restriction on $w$ implied by this scalar field realization is that it must lie in the interval $[-1,1]$. However, we have found, by requiring this spacetime to arise from a cosmological compactification of a higher-dimensional gravitational theory with stress tensor satisfying the SEC, that

$$
\eta<\eta_{\max }<4 / 3
$$

where the second inequality, which is independent of both $d$ and $D$, translates to the following lower bound on $w$ :

$$
w>-1+\frac{3}{2(d-1)} .
$$

In subsection 2.2 we concluded (based on assumptions stated there) that a dS universe cannot be obtained by compactification even if the compact space and warp factor are allowed to be time dependent. This conclusion (along with the assumptions on which it depends) is in agreement with earlier work on a generalization of the GMN no-go theorem applying to time-independent compactifications. In the current context, this generalized no-go theorem could be rephrased as the statement that $w=-1$ is excluded, but we have now arrived (under the same assumptions) at a much stronger restriction on $w$; for $d=4$ this restriction is the lower bound $w>-1 / 2$. Of course, this statement applies to a perfect fluid with an equation of state $P=w \rho$ for constant $w$, which is (unless $w=-1$ ) equivalent to the assumption of power-law expansion, which we can only expect to be valid at late times.

\subsection{The Dominant Energy Condition}

The SEC on the $D$-dimensional stress tensor is

$$
(D-3) \rho_{0}+(d-1) P_{0}+n P_{0}^{(\mathrm{int})} \geq 0 .
$$

As a check one may use the Einstein equations in the form $(3.13)$ and the expressions of (3.9) for $(A, B, C)$ to show that this implies the inequality 2.22 . As we have seen, this inequality is compatible with acceleration, i.e. with $\eta>1$, but for any such choice of $(\eta, \xi)$ we need to check that the DEC is satisfied. This condition states that no component of the stress tensor may have a magnitude greater than the energy density, which must be non-negative. In the present context, this is equivalent to

$$
\rho_{0} \geq 0, \quad \rho_{0} \pm P_{0} \geq 0, \quad \rho_{0} \pm P_{0}^{(\text {int })} \geq 0,
$$

for any choice of the signs. As we already know that $\rho_{0}>0$ and that both $P_{0}$ and $P_{0}^{\text {(int) }}$ are negative when the SEC is satisfied, we only need to check positivity of $\rho_{0}+P_{0}$ and $\rho_{0}+P_{0}^{(\text {int })}$. We consider them in turn: 
- $\rho_{0}+P_{0}$. In this case we have from (3.14) that

$$
\begin{aligned}
\rho_{0}+P_{0} & =(d-2)\left[\eta-\frac{(D-2)}{n} \xi^{2}\right] \\
& >(d-2)\left[1-\frac{n}{(D-2)}\right]=\frac{(d-2)^{2}}{D-2}>0,
\end{aligned}
$$

where the inequality follows from $\eta>1$ and $|\xi|<n /(D-2)$.

- $\rho_{0}+P_{0}^{(\mathrm{int})}$. In this case it is simplest to solve the linear relation 3.12 for $P_{0}$ in terms

of $\rho_{0}$ and $P_{0}^{\text {(int) }}$, and then substitute the result into the SEC condition 3.24). This yields the following inequality:

$$
[n \eta+(D-2) \xi]\left(\rho_{0}+P_{0}^{(\mathrm{int})}\right) \geq 2(\eta-1) \rho_{0} .
$$

The right hand side is positve for $\eta>1$. The coefficient $[n \eta+(D-2) \xi]$ is also positive for $\eta>1$ as a consequence of the bounds on $\xi$ imposed by the SEC. We thus conclude that

$$
\rho_{0}+P_{0}^{(\text {int })}>0,
$$

when $\eta>1$, as a consequence of the SEC.

This concludes our demonstration, for the specific power-law type cosmological compactification under consideration, that the DEC is a consequence of the SEC when the lowerdimensional FLRW universe is undergoing accelerated expansion. Taken together with our finding that the SEC conditions are compatible with acceleration, we conclude that the combined SEC and DEC conditions in the higher dimension do not exclude the possibility of compactification to an eternally accelerating FLRW universe (to which other solutions could asymptote at late time).

\subsection{Asymptotic zero acceleration}

As mentioned earlier, the $\eta=1$ case of the power-law solutions considered above should be taken into account in any discussion of late-time acceleration because a universe expanding with zero acceleration may be approached asymptotically by one that has non-zero positive acceleration at late times. Allowing for $\eta=1$ in the above analysis so far does not change the conclusions, as long as $k=0$; i.e. as long as the $(d-1)$-space has zero curvature. However, setting $\eta=1$ in the Ricci tensor expressions of (3.2) and (3.6) yields

$$
\begin{aligned}
R_{00} & =-\frac{(d-2)}{t^{2}} \xi\left[1+\frac{(D-2)}{n} \xi\right], \\
R_{i j} & =(1+k+\xi)(d-2) \bar{g}_{i j}, \\
R_{\alpha \beta} & =-\frac{(d-2)^{2}}{n} t^{-2(1+\xi)} \xi h_{\alpha \beta} .
\end{aligned}
$$


The space curvature $k$ is now relevant.

We consider here only the vacuum Einstein equations. These have a solution for $k=$ -1 , provided that $\xi=0$, which implies that the metric on the compact space is timeindependent; this is the compactification to a Milne universe discussed in [26]. In general, one may expect there to exist initial conditions that lead to accelerating universes that are asymptotic, at late time, to a zero-acceleration universe; a special feature of this case is that, despite the late time acceleration, there is no future cosmological event horizon [27]. Examples were found in [28] for cosmological models with "double exponential potentials" (for which exact solutions may also be found [29]).

\subsection{Generic Einstein metric for the compact space}

So far we supposed the metric $h$ on the compact space $B$ to be Ricci-flat. More generally, we could suppose that it is an Einstein metric, in which case $\tilde{R}_{\alpha \beta}=(n-1) K \tilde{h}_{\alpha \beta}$ for constant $K$. In this case, the expression of $(3.6)$ is replaced by

$$
R_{\alpha \beta}=-t^{-2(1+\xi)}\left\{[(d-2) / n][(d-1) \eta-1] \xi-t^{2\left[1+\frac{(D-2)}{n} \xi\right]}(n-1) K\right\} h_{\alpha \beta} .
$$

Let us define

$$
\xi_{0}=-\frac{n}{(D-2)}
$$

and consider in turn the implications of choosing $\xi$ to be less than, greater than or equal to $\xi_{0}$ :

- $\xi<\xi_{0}$. The curvature term proportional to $K$ is subleading at late times and can be ignored, so we are back to the $K=0$ case.

- $\xi>\xi_{0}$. The curvature term proportional to $K$ dominates and there is no vacuum solution of the assumed power-law form.

- $\xi=\xi_{0}$. In this case the SEC, which is unaffected by the curvature of space, requires

$$
\eta^{2}-\left(1+\frac{n}{(D-2)}\right) \eta+\frac{n}{(D-2)} \leq 0 \Rightarrow\left|\xi_{0}\right| \leq \eta \leq 1 .
$$

Only $\eta=1$ (which implies saturation of the SEC bound) is relevant to the possibility of late-time acceleration, and in this case we should also allow for non-zero $k$. Again restricting to vacuum solutions (i.e. all components of the Ricci tensor are zero), we find that there is just one of them, provided that we choose (recall that $k$ is un-normalized)

$$
k=-\frac{(d-2)}{(D-2)}, \quad K=-\frac{(d-2)^{2}}{(D-2)(n-1)} .
$$

We remark that the SEC is also saturated for $\xi=\xi_{0}$ when $\eta=n /(D-2)$. In this case, $\eta<1$ and there is a vacuum solution for $K=-(d-2)^{2} /(D-2)^{2}$ provided that $k=0$. This decelerating scaling solution is the late-time limit of the hyperbolic compactification exhibiting transient cosmic acceleration found in [11]. 


\section{Summary and discussion}

We have explored the constraints on cosmological compactifications implied by the strong energy condition (SEC) on the higher-dimensional stress tensor. It is known that this condition rules out compactification to de Sitter space (dS) if the compact space is non-singular and time-independent. This no-go theorem follows from a simple inequality implied by the SEC on the scale factor of de Sitter space viewed as an FLRW universe. A new result of this paper is the corresponding inequality (2.13) for compactification to any Einsteinframe FLRW universe, allowing for any time dependence compatible with generic FLRW isometries. However, this inequality does not lead directly to any interesting conclusions in particular, it does not obviously rule out compactifications to de Sitter. This is mainly because the Einstein-frame condition involves an averaging over the compact space. In known examples the Einstein-frame condition is satisfied in an unaveraged form, and if this is assumed the SEC inequality simplifies dramatically to the form $(2.19)$; the inequality used in [9] is then found by ignoring terms involving first derivatives, but their inclusion is essential to our analysis of the implications of the SEC for late-time behaviour.

Many examples are known of time-dependent compactifications of $D=10 / 11$ supergravity theories for which there is a period of acceleration, in Einstein frame, but the late time universe in all these examples is decelerating. Since these supergravity theories satisfy the SEC, this might be a consequence of the SEC. However, we have shown, by example, that the SEC, by itself, does not prevent compactification to (Einstein frame) universes that undergo late-time accelerated expansion. Our examples are scaling solutions that represent a time-dependent compactification to an FLRW universe that undergoes an eternal accelerated expansion (and hence, in particular, at late times) but we expect these to act as late-time attractor solutions for more general FLRW compactifications. To verify this we would first need a better understanding of the $D$-dimensional stress tensor that makes such compactifications possible; at present we know only its late-time form, which suggests some anisotropic generalization of a perfect fluid.

What we can say of our examples is that they are compactifications to FLRW universes filled with a perfect fluid that has a constant pressure to energy density ratio $w$, on which the higher dimensional SEC imposes a lower bound, which is $w>-1 / 2$ for $d=4$. The constancy of $w$ is implied by the power-law expansion, which is accelerated expansion when (for $d=4) w<-1 / 3$. This shows, in particular, that the SEC can be violated in the lower dimension even though it is not violated in the higher dimension. We should stress that the lower bound on $w$ (which excludes the $\mathrm{dS}$ case of $w=-1$ ) is a late-time result since we can expect $w$ to be constant only at late times (and then only if $w<-1 / 3$ ). There is therefore no conflict with the fact that observations are consistent with a present value of $w=-1$, especially as they are also consistent with increasing $w[20$.

Another feature of our examples is that the compact space is also expanding, which implies a late-time decompactification; in practice this could be defined to occur at a decompactification time $T$ at which the effects of massive Kaluza-Klein (KK) particles become apparent in accelerator experiments. There could then be a conflict with observations if $T$ is less than the current age of our Universe. However, a remarkable property of our exam- 
ples is that accelerated expansion of the FLRW universe requires a decelerating expansion of the compact space. Assuming that the lightest KK particle would become observable when its mass decreases to the Higgs mass, we have shown that the decompactification time is arbitrarily large and, generically, much longer than the current age of our Universe.

\subsection{No-acceleration bounds on exponential scalar potentials}

An alternative response to our results would be to ask why we should have expected to extract so much from the SEC, which has no obvious fundamental significance. Quite possibly, our examples of cosmic late-time acceleration from compactification cannot be realized in string/M-theory for other reasons. Or it could be ruled out by other general considerations, such as those mentioned in the introduction. As also mentioned there, the proposed cosmological swampland bound of $[9$ is of the type that can be easily deduced for positive exponential potentials in one-scalar theories if one supposes that late-time acceleration is impossible. As promised in the Introduction, we conclude with a comment on this bound.

For a one-scalar theory with a positive exponential potential $V$, there will be cosmological solutions that undergo accelerated expansion at late time unless $|\nabla V| / V>c$, with $c=\mathcal{O}(1)$, which is the swampland bound proposed in [9]. However, the situation is very different for a two-scalar model, even for a positive exponential potential that is a function of only one of the two fields; in this case the no-acceleration bound on the coefficient $V^{\prime} / V$ can depend on the interaction between the two scalar fields.

An example is provided by the following Lagrangian for a dilaton field $\sigma$ and an axion field $\chi$ whose vacuum values parametrize the hyperbolic space $H_{2} \cong S l(2 ; R) / S O(2)$ :

$$
L=R-\frac{1}{2}\left[(\partial \sigma)^{2}+e^{-\mu \sigma}(\partial \chi)^{2}\right]-\Lambda e^{-\lambda \sigma} .
$$

We may assume that $\lambda>0$ but then $\mu$ could be positive or negative; its absolute value is inversely proportional to the radius of curvature of the $H_{2}$ target space. We also assume that $\Lambda>0$, since accelerating cosmologies are otherwise not possible. For constant $\chi$ there is a scaling solution of the equations for expanding FLRW cosmologies and the expansion is accelerating if $\lambda<\lambda_{c}$ where $\lambda_{c}$ is a 'critical' value. For a $d$-dimensional spacetime, and in our conventions ${ }^{6}$,

$$
\lambda_{c}=\sqrt{\frac{2}{d-2}},
$$

so that $\lambda_{c}=1$ for $d=4$.

However, there is also a scaling scaling solution with non-constant $\chi$ if $\mu>0$ and in this case the scale factor (in terms of standard FLRW time $t$ ) is [30]

$$
S \propto t^{\eta}, \quad \eta=\frac{\lambda+\mu}{(d-1) \lambda} .
$$

\footnotetext{
${ }^{6}$ Given in detail in 30 except that here we have flipped the sign of $\mu$.
} 
Accelerated expansion occurs when $\eta>1$, which is equivalent to

$$
\lambda<\frac{\mu}{d-2} .
$$

The right hand side is a new critical value for $\lambda$, which can be larger than $\lambda_{c}$ for sufficiently large $\mu$.

To avoid a possible late-time acceleration in this dilaton-axion model we must impose both $\lambda>\lambda_{c}$ and $\mu<(d-2) \lambda$; given $\lambda>\lambda_{c}$, a sufficient condition on $\mu$ is

$$
\mu<\mu_{c}=\sqrt{2(d-2)},
$$

so $\mu_{c}=2$ for $d=4$. This is a weak-coupling requirement. The stronger the coupling the more damped is the rolling down the exponential potential due to transfer of potential energy to the axion, which raises the critical value of $V^{\prime} / V$ corresponding to a crossover from deceleration to acceleration. The physics is analogous to the rolling of a disc of large moment of inertia down a hill under the influence of gravity; potential energy is transferred not only to kinetic energy of downward motion but also to rotational kinetic energy, which slows the downward motion.

\section{Acknowledgments}

We ae grateful to Thomas Van Riet for helpful correspondence. JGR acknowledges financial support from projects 2017-SGR-929, MINECO grant FPA2016-76005-C. The work of PKT has been partially supported by STFC consolidated grant ST/L000385/1.

\section{References}

[1] E. Witten, "Quantum gravity in de Sitter space," hep-th/0106109.

[2] S. Hellerman, N. Kaloper and L. Susskind, "String theory and quintessence," JHEP 0106 (2001) 003 [hep-th/0104180].

[3] W. Fischler, A. Kashani-Poor, R. McNees and S. Paban, "The Acceleration of the universe, a challenge for string theory," JHEP 0107 (2001) 003 hep-th/0104181.

[4] G. W. Gibbons, "Aspects Of Supergravity Theories," in Supersymmetry, Supergravity and Related Topics, eds. F. Aguila, J.A. de Azcárraga, and L. Ibañez, (World Scientific, 1985) [DAMTP preprint 85-0061]; "Thoughts on tachyon cosmology," Class. Quant. Grav. 20 (2003) S321 hep-th/0301117.

[5] J. M. Maldacena and C. Nuñez, "Supergravity description of field theories on curved manifolds and a no go theorem," Int. J. Mod. Phys. A 16 (2001) 822 [hep-th/0007018]. 
[6] S. Kachru, R. Kallosh, A. D. Linde and S. P. Trivedi, "De Sitter vacua in string theory," Phys. Rev. D 68 (2003) 046005 [hep-th/0301240].

[7] M. Cicoli, S. De Alwis, A. Maharana, F. Muia and F. Quevedo, "De Sitter vs Quintessence in String Theory," Fortsch. Phys. 20181800079 arXiv:1808.08967 [hepth]].

[8] U. H. Danielsson and T. Van Riet, "What if string theory has no de Sitter vacua?," Int. J. Mod. Phys. D 27 (2018) no.12, 1830007 arXiv:1804.01120 [hep-th]].

[9] G. Obied, H. Ooguri, L. Spodyneiko and C. Vafa, "De Sitter Space and the Swampland," arXiv:1806.08362 [hep-th].

[10] E. Teo, "A No-go theorem for accelerating cosmologies from M-theory compactifications," Phys. Lett. B 609 (2005) 181 [hep-th/0412164].

[11] P. K. Townsend and M. N. R. Wohlfarth, "Accelerating cosmologies from compactification," Phys. Rev. Lett. 91 (2003) 061302 [hep-th/0303097].

[12] L. Cornalba and M. S. Costa, "A new cosmological scenario in string theory," Phys. Rev. D 66 (2002) 066001 [hep-th/0203031].

[13] N. Ohta, "Accelerating cosmologies from S-branes," Phys. Rev. Lett. 91 (2003) 061303 hep-th/0303238; " A Study of accelerating cosmologies from superstring/M theories," Prog. Theor. Phys. 110 (2003) 269 [hep-th/0304172].

[14] S. Roy, "Accelerating cosmologies from M/string theory compactifications," Phys. Lett. B 567 (2003) 322 hep-th/0304084.

[15] M. N. R. Wohlfarth, "Accelerating cosmologies and a phase transition in M theory," Phys. Lett. B 563 (2003) 1 hep-th/0304089.

[16] C. M. Chen, P. M. Ho, I. P. Neupane and J. E. Wang, "A Note on acceleration from product space compactification," JHEP 0307 (2003) 017 hep-th/0304177.

[17] M. Gutperle, R. Kallosh and A. D. Linde, "M/string theory, S-branes and accelerating universe," JCAP 0307 (2003) 001 hep-th/0304225.

[18] P. K. Townsend, "Quintessence from M-theory," JHEP 0111 (2001) 042 hepth/0110072.

[19] R . Emparan and J. Garriga, "A Note on accelerating cosmologies from compactifications and S branes," JHEP 0305 (2003) 028 [hep-th/0304124].

[20] N. Aghanim et al. [Planck Collaboration], "Planck 2018 results. VI. Cosmological parameters," arXiv:1807.06209 [astro-ph.CO]. 
[21] P. G. Ferreira and M. Joyce, "Cosmology with a primordial scaling field," Phys. Rev. D 58 (1998) 023503 astro-ph/9711102.

[22] J. G. Russo, "Exact solution of scalar tensor cosmology with exponential potentials and transient acceleration," Phys. Lett. B 600 (2004) 185 [hep-th/0403010].

[23] P. K. Townsend, "Cosmic acceleration and M-theory," in proceedings of the 14th International Congress on Mathematical Physics (ICMP03), ed. J.C. Zambrini (World Scientific 2006); [hep-th/0308149].

[24] P. K. Townsend and M. N. R. Wohlfarth, "Cosmology as geodesic motion," Class. Quant. Grav. 21 (2004) 5375 [hep-th/0404241].

[25] J. Borlaf, "Perfect fluids of p-branes from theories invariant under field redefinitions," Phys. Rev. D 60 (1999) 046001.

[26] G. W. Gibbons and P. K. Townsend, "Cosmological Evolution of Degenerate Vacua," Nucl. Phys. B 282 (1987) 610.

[27] L. J. Boya, M. A. Per and A. J. Segui, "Graphical and kinematical approach to cosmological horizons," Phys. Rev. D 66 (2002) 064009 [gr-qc/0203074.

[28] L. Jarv, T. Mohaupt and F. Saueressig, "Quintessence cosmologies with a double exponential potential," JCAP 0408 (2004) 016 [hep-th/0403063].

[29] N. Kan, M. Kuniyasu, K. Shiraishi and K. Takimoto, "Integrable higher-dimensional cosmology with separable variables in an Einstein-dilaton-antisymmetric field theory," Phys. Rev. D 98 (2018) no.4, 044054 arXiv:1806.10263 [hep-th]].

[30] J. Sonner and P. K. Townsend, "Recurrent acceleration in dilaton-axion cosmology," Phys. Rev. D 74 (2006) 103508 [hep-th/0608068]. 